\title{
Reliability of an Observational Biomechanical Analysis Tool in Adolescent Baseball Pitchers
}

\author{
Steven F DeFroda, MD', Dai Sugimoto, PhD², Steven J Staffa, $\mathrm{MS}^{3}$, Donald S Bae, MDㄴ, Ellen Shanley, PhD, PT, OCS ${ }^{5}$, \\ Charles A Thigpen, PhD, $\mathrm{PT}^{5}$, Peter K Kriz, MD ${ }^{\text {a }}$ \\ ${ }^{1}$ Departments of Orthopedics, Warren Alpert Medical School, Brown University, ${ }^{2}$ The Micheli Center for Sports Injury Prevention; Division of Sports \\ Medicine, Department of Orthopaedics, Boston Children's Hospital, Boston; Department of Orthopaedic Surgery, Harvard Medical School, 3 \\ Department of Anesthesiology, Critical Care and Pain Medicine, Boston Children's Hospital, ${ }^{4}$ Division of Sports Medicine, Department of \\ Orthopaedics, Boston Children's Hospital, 5 ATI Physical Therapy; Hawkins Foundation; Center for Effectiveness Research in Orthopedics \\ Keywords: analysis, pitching mechanics, throwing athlete \\ https://doi.org/10.26603/001c.29869
}

\section{International Journal of Sports Physical Therapy}

Vol. 16, Issue 6, 2021

\section{Background}

Improper pitching mechanics are a risk factor for arm injuries. While 3-dimensional (3D) motion analysis remains the gold standard for evaluation, most pitchers and clinicians do not have access to this costly technology. Recent advances in 2-dimensional (2D) video technology provide acceptable resolution for clinical analysis. However, no systematic assessment tools for pitching analysis exist.

\section{Purpose}

To determine the reliability of the Assessment of biomeChanical Efficiency System (ACES) screening tool using 2D video analysis to identify common biomechanical errors in adolescent pitchers.

\section{Study Design}

Cross-sectional.

\section{Methods}

Adolescent baseball pitchers underwent analysis using 2D video in indoor settings. Observational mechanics were collected using a 20-item scoring tool (ACES) based on 2D video analysis. Fleiss' kappa, interclass correlation coefficients (ICC), and frequencies were used to examine intra-/interrater reliability based on common pitching errors.

\section{Results}

Twenty asymptomatic pitchers ages 12-18 years were included. Total ACES scores ranged from 1 to 13, normally distributed. ACES total score demonstrated excellent intra-rater reliability within each rater (ICC for rater $1=0.99$ (95\% CI; 0.98, 0.99); ICC for rater $2=$ 0.94; 95\% CI: 0.84, 0.97); ICC for rater $3=0.98$ (95\% CI: 0.96, 0.99)). There was excellent interrater reliability across the trials and raters (ICC $=0.91 ; 95 \%$ CI: $0.82,0.96$ ). The ACES tool demonstrated acceptable kappas for individual items and strong ICC 0.91 (95\% CI: $0.82,0.96)$ for total scores across the trials. Regarding identification of biomechanical errors, "front side position" was rated erroneous in 84/120 ratings (70\%), stride length in $52 / 120$ ratings (43.3\%) and lead hip position in 53/120 ratings (44.2\%).

\section{Conclusions}

The 20-item ACES scoring tool with 2D video analysis demonstrated excellent intra- and interrater reliability when utilized by raters of different musculoskeletal disciplines. Future studies validating 2D vs. 3D methodology are warranted before ACES is widely disseminated and utilized for adolescent pitchers. ACES is a practical and reliable clinical

\footnotetext{
a Corresponding Author:

Kettle Point Avenue, East Providence, RI, 02915, (401) 457-2188 phone, (401) 457-2187 fax, Peter_Kriz@brown.edu
} 
assessment tool utilizing 2D video analysis for coaches, instructors, and sports medicine providers to screen adolescent pitchers for common biomechanical errors.

\section{Level of Evidence}

$3 \mathrm{~b}$

\section{INTRODUCTION}

Upper extremity injuries in youth and adolescent baseball pitchers remain common, ranging from self-limited, growth-related disturbances including Little League Shoulder to career-threatening injuries such as ulnar collateral ligament (UCL) tears. ${ }^{1-8}$ Risk factors for injury are multifactorial and include overuse, fatigue, player demographics, throwing volume and velocity, improper biomechanics, and kinetic chain imbalances pertaining to strength, range of motion, and flexibility.3,5,9-11 Technological advances in 3-dimensional (3D) motion analysis and high-speed videography in the past few decades have contributed to a better understanding of pitching biomechanics in pitchers of all ages and levels. ${ }^{12,13}$ While the preponderance of research has focused on the collegiate and professional pitcher, recent investigations of youth and adolescent pitching mechanics have led to a refined comprehension of critical relationships between functional strength, mobility, and stability as they relate to mechanical efficiency, injury risk, and performance. ${ }^{14-18}$ Despite these technological advances, pitching-related youth and adolescent upper extremity injuries have reached epidemic proportions. ${ }^{5,19,20}$ Ulnar collateral ligament reconstruction (the "Tommy John surgery") now has a higher incidence in 15 to 19 year-old throwing athletes than any other group, including professional athletes. ${ }^{19}$

The gold standard for assessing pitching biomechanics in all age groups is 3D motion analysis, which provides valuable quantitative measures of pitching kinetics and kinematics; however, it is costly, time- and resource-intensive, typically limited to a single-episode analysis in a laboratory setting, and not accessible to all socioeconomic levels. ${ }^{12-18,21}$ Consequently, there is a critical need to develop an evidence-based screening tool that can be utilized by a diverse group of users, including coaches, instructors, and sports medicine providers to identify youth and adolescent pitchers with at-risk mechanics and strength/flexibility deficits early in their development. Qualitative 2-dimensional (2D) video analysis, in conjunction with observational measurements, has the potential to be a practical, cost-effective clinical assessment. ${ }^{21}$ The "Assessment of biomeChanical Efficiency System" (ACES) is an observational measurement tool that was designed by the authors to evaluate pitching mechanics in young baseball pitchers. The ACES consists of 20 scoring items that are rated by $2 \mathrm{D}$ video analysis. Although the ACES is an evidence-based scoring system (Appendix I), its reliability has not been assessed; therefore, the purpose of this study was to evaluate the reliability of the ACES screening tool using 2D video analysis to identify common biomechanical errors in adolescent pitchers. We hypothesized that the ACES tool would demonstrate good intra-rater and inter-rater reliability for identifying biomechanical errors in adolescent pitchers.

\section{METHODS}

\section{PARTICIPANTS}

Middle school and high school male pitchers with a minimum of two years' pitching experience were recruited from local high schools and from the study institutions' sports medicine clinics. Pitchers were males between the ages of 12 and 18 years old. Pitchers with any current injury that affected normal mechanics were excluded. Sidearm or submarine pitchers, defined as pitchers who maintain their pitching arm in a low, approximately horizontal plane (eg, at or below the 9 o'clock position for right-handed pitchers, 3 o'clock position for left-handed pitchers as illustrated in Appendix II), were also excluded from the study.

\section{INSTRUMENTATION/DATA COLLECTION}

Testing occurred at two different locations (Rhode Island Hospital and Boston Children's Hospital.) Following a proper warm-up, all participants performed 10 to 25 fastball pitches using high-speed 2D video analysis. The primary investigator (PKK) reviewed all participants' videos and selected the one pitch that represented their best effort and fastball mechanics for analysis; kinematic variables were then assessed using 2D motion analysis software (Dartfish Inc.; Alpharetta, GA) with commercially available high-resolution video cameras (GoPro Hero 3, 120 frames per second, San Mateo, CA; Casio Exilim Pro EX-F1, 300 frames per second, Tokyo, Japan) recording from frontal and lateral views. Pitchers threw off an indoor mound (ProMounds; Chatsworth, GA) to a catcher (at Rhode Island Hospital) or to a strike zone target (at Boston Children's Hospital) at a distance commensurate with their level of play. Visual assessment of the upper extremities, lower extremities, trunk, and pelvis was collected and computed through each phase of the pitching cycle (Figure 1).

Raters used a scoring system (ACES) to assess 20 kinematic variables and observational measurements that were identified by biomechanics researchers to be key features of the pitching cycle ${ }^{13}$ (Appendices I-III), as well as an overall impression of the thrower's mechanics. Observational measurements were recorded by each rater for the 20 kinematic variables in a binary fashion ( $1=$ error; $0=$ no error). One point was given for each error in the throwing sequence, with a perfect score being 0 and the worst possible score is 21 .

Pitchers' videos were examined by three raters from different clinical sports medicine specialties (orthopedic surgery resident, physical therapist, and athletic trainer) with varying experience analyzing youth pitching mechanics. All raters participated in a 30-minute training webinar and completed five reviews together prior to study assessments. The ACES tool was used to assess the videos twice, with a washout period of two weeks between rater assessments. The research team intentionally chose raters with 


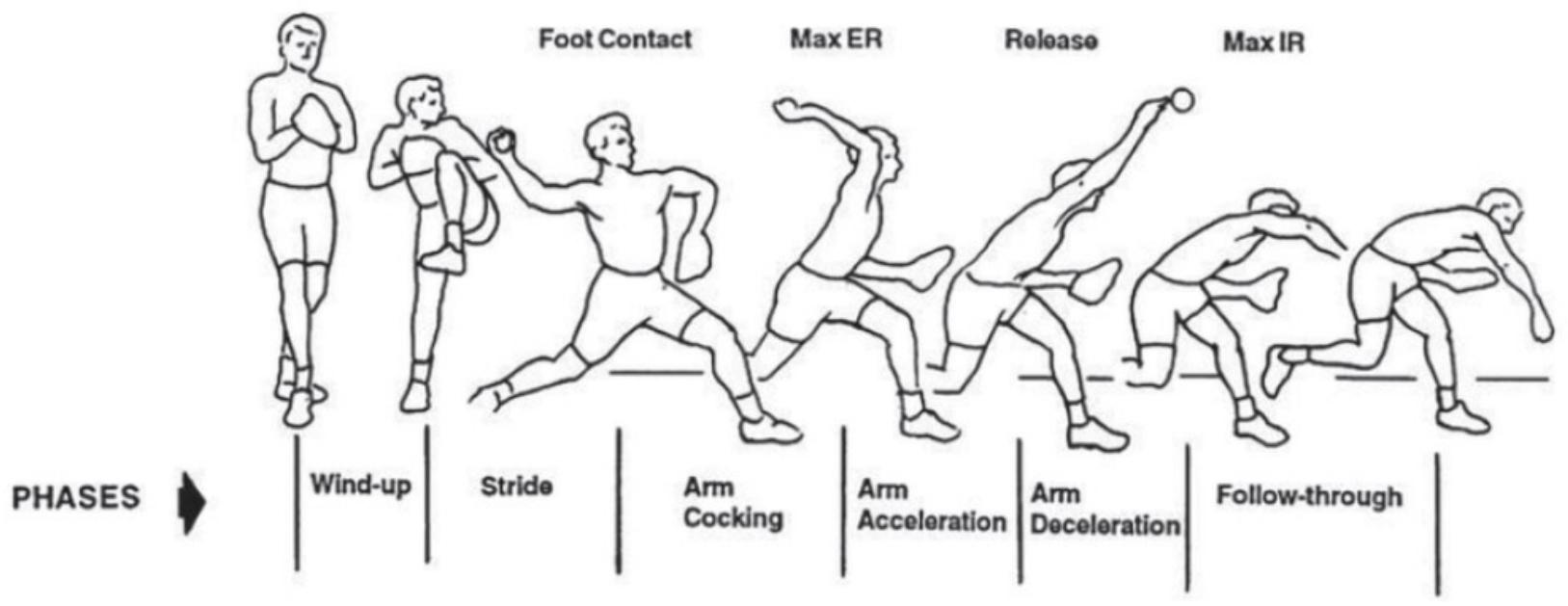

\section{Figure 1. Phases of The Overhand Throw}

Reprinted from Journal of Biomechanics, Vol 32, Fleisig GS, Barrentine SW, Zheng N, Escamilla RF, Andrews JR. Kinematic and kinetic comparison of baseball pitching among various levels of development, 1371-1375, Copyright (1999), with permission from Elsevier.

different career backgrounds because the scoring system was developed for use by a heterogenous group of evaluators (eg, coaches, instructors, and healthcare providers). This cross-sectional study was approved by the Institutional Review Boards of Rhode Island Hospital and Boston Children's Hospital.

\section{STATISTICAL ANALYSIS}

Data on individual items were summarized as frequencies and percentages across all ratings of pitchers by the three raters. Total scores for each rater were summarized as mean and standard deviation and range. Absolute agreement (\%) and Fleiss' kappa $(\kappa)$ coefficient were calculated to determine intra- and inter-rater reliability of pitching errors for the individual binary items. Intraclass correlation coefficients (ICC) were calculated to determine intra- and inter-rater agreement on total scores. The ICC values were calculated using two-way random effects modeling (Stata Statistical Software: Release 16; College Station, TX) because the same set of raters scored all the pitchers. Agreement was determined based on established interpretations criteria: ${ }^{22,23}$ poor agreement $(<0.4)$, fair to good agreement (0.4 to 0.75$)$, and excellent agreement $(\geqslant 0.75)$.

\section{RESULTS}

Twenty asymptomatic male pitchers (mean age $15.1 \pm 3.1$ years old) participated in the study. The ACES total scores ranged from 1 to 13 and were normally distributed with mean scores across raters of $7.5 \pm 3.0 ; 6.7 \pm 2.3$; and $5.5 \pm$ 2.6, respectively. The ACES total score demonstrated excellent intra-rater reliability within each rater (ICC for rater $1=0.99$ (95\% CI; 0.98, 0.99); ICC for rater $2=0.94 ; 95 \%$ CI: 0.84, 0.97); ICC for rater $3=0.98$ (95\% CI: 0.96, 0.99)). There was excellent inter-rater reliability across the trials and raters (ICC $=0.91$; 95\% CI: 0.82, 0.96). For individual items, absolute inter-rater agreement across the three raters ranged from $50 \%$ to $100 \%$ (Table 1). Intra-rater con- sistency of individual items was strong, with absolute agreement ranging from $70 \%$ to $100 \%$, and kappa values from 0.38 to 1 (Table 1 ).

Various items were most often recorded as erroneous by the three raters. Specifically, ACES item 10 (Appendix III) which is designated "front side position" in Table 1 and Figure 2, was rated erroneous in 84 of 120 ratings (70\%).

ACES item 9, stride length, was rated erroneous in 52 of 120 ratings (43.3\%). Lead hip position, ACES item 3 during the windup phase, also accounted for many errors (53 in 120 ratings, or $44.2 \%$ ).

\section{DISCUSSION}

The purpose of this study was to determine the reliability of a practical, affordable clinical assessment tool utilizing $2 \mathrm{D}$ video analysis for coaches, instructors, and sports medicine providers to screen adolescent pitchers for common biomechanical errors. The ACES scoring tool demonstrated excellent reliability for identifying biomechanical errors in adolescent pitchers when administered by raters of different musculoskeletal disciplines. Regarding each ACES item, there was a diverse range of scores; however, acceptable values were found by weighted kappa analysis overall.

Previous authors have analyzed qualitative kinematic variables during the pitching cycle in adolescent baseball players. Nicholls et al. ${ }^{24}$ evaluated 20 adolescent baseball pitchers using qualitative $2 \mathrm{D}$ and 3D motion analysis to validate kinematic variables from the stride, arm cocking, and arm acceleration/ball release phases. Two raters (an internationally recognized coach and a trained biomechanist) conducted an analysis using a 24-item qualitative analysis protocol of ideal pitching techniques. Intra-rater reliability across two trials was fair to excellent, with all 24 items in the checklist producing kappa between 0.400 and 0.900 (in 22 items, $\mathrm{p}<0.05$ ). Inter-rater reliability for the qualitative analysis protocol was fair to good (kappa $=0.468$ to 0.694 , $\mathrm{p}<0.05$ ) on $8 / 24$ (33\%) qualitative kinematic variables: four stride phase variables (stride offset, foot angle, shoulder ex- 


\begin{tabular}{|c|c|c|c|c|c|}
\hline \multirow[b]{2}{*}{ ACES Item } & \multirow[b]{2}{*}{$\begin{array}{l}\text { Number with } \\
\text { condition error } \\
\text { among all } 120 \\
\text { ratings }(\%)\end{array}$} & \multicolumn{2}{|c|}{$\begin{array}{l}\text { Inter-rater reliability (between } \\
\text { raters) }\end{array}$} & \multicolumn{2}{|c|}{ Intra-rater reliability (within raters) } \\
\hline & & $\begin{array}{l}\text { Absolute } \\
\text { agreement } \\
\text { across raters } \\
\text { (Rating 1/ } \\
\text { Rating 2) }\end{array}$ & $\begin{array}{c}\text { Fleiss' } \\
\text { kappa }{ }^{\star *} \\
\text { (Rating 1 / } \\
\text { Rating 2) }\end{array}$ & $\begin{array}{c}\text { Absolute agreement } \\
\text { within each rater } \\
\text { (Rater 1/ Rater 2 / } \\
\text { Rater 3) }\end{array}$ & $\begin{array}{c}\text { Fleiss' } \\
\text { kappa** } \\
\text { (Rater 1 / } \\
\text { Rater 2 / } \\
\text { Rater 3) }\end{array}$ \\
\hline \multicolumn{6}{|l|}{ Windup } \\
\hline Center of Gravity & $37(30.8 \%)$ & $78 \% / 80 \%$ & $0.44 / 0.54$ & $100 \% / 95 \% / 100 \%$ & $1 / 0.89 / 1$ \\
\hline Knee Height & $22(18.3 \%)$ & $93 \% / 93 \%$ & $0.78 / 0.78$ & $100 \% / 100 \% / 100 \%$ & $1 / 1 / 1$ \\
\hline Lead IIip & $53(44.2 \%)$ & $77 \% / 67 \%$ & $0.53 / 0.12$ & $100 \% / 85 \% / 100 \%$ & $1 / 0.70 / 1$ \\
\hline \multicolumn{6}{|l|}{ Stride } \\
\hline Hands Separation/Position & $28(23.3 \%)$ & $73 \% / 70 \%$ & $0.29 / 0$ & $100 \% / 95 \% / 95 \%$ & $1 / 0.83 / 0.89$ \\
\hline Hip Rotation & $13(10.8 \%)$ & $87 \% / 77 \%$ & $0.13 / 0$ & $100 \% / 85 \% / 100 \%$ & $1 / 0.63 / 1$ \\
\hline Ilands on Top & $24(20 \%)$ & $70 \% / 77 \%$ & $0.06 / 0.27$ & $100 \% 85 \% / 95 \%$ & $1 / 0.66 / 0.83$ \\
\hline Time to Lead Foot & $8(6.7 \%)$ & $87 \% / 87 \%$ & $-0.07 /-0.07$ & $100 \% / 100 \% / 100 \%$ & $1 / 1 / 1$ \\
\hline \multicolumn{6}{|l|}{ Stride Foot Contact } \\
\hline External Rotation at SFC & $38(31.7 \%)$ & $80 \% / 77 \%$ & $0.52 / 0.48$ & $100 \% / 90 \% / 100 \%$ & $1 / 0.78 / 1$ \\
\hline Stride Length & $52(43.3 \%)$ & $73 \% / 73 \%$ & $0.46 / 0.46$ & $100 \% / 100 \% / 100 \%$ & $1 / 1 / 1$ \\
\hline Front Side Position & $84(70 \%)$ & $73 \% / 70 \%$ & $0.38 / 0.26$ & $100 \% / 95 \% / 95 \%$ & $1 / 0.90 / 0.86$ \\
\hline Trunk Rotation & $44(36.7 \%)$ & $83 \% / 77 \%$ & $0.64 / 0.5$ & $95 \% / 85 \% / 100 \%$ & $0.89 / 0.66 / 1$ \\
\hline \multicolumn{6}{|l|}{ Arm Cocking } \\
\hline Trunk Tilt & $36(30 \%)$ & $57 \% / 50 \%$ & $-0.07 /-0.16$ & $100 \% / 80 \% / 100 \%$ & $1 / 0.60 / 1$ \\
\hline External Rotation at Max Cocking & $7(5.8 \%)$ & $90 \% / 93 \%$ & $0.2 / 0.3$ & $100 \% / 95 \% / 100 \%$ & $1 / 0.61 / 1$ \\
\hline \multicolumn{6}{|l|}{ Acceleration } \\
\hline Trunk Flexion & $24(20 \%)$ & $83 \% / 83 \%$ & $0.48 / 0.18$ & $100 \% / 90 \% / 100 \%$ & $1 / 0.69 / 1$ \\
\hline Knee Flexion & $26(21.7 \%)$ & $90 \% / 90 \%$ & $0.71 / 0.71$ & $100 \% / 80 \% / 100 \%$ & $1 / 0.47 / 1$ \\
\hline \multicolumn{6}{|l|}{ Deceleration } \\
\hline Arm Internal Rotation & $0(0 \%)$ & $100 \% / 100 \%$ & $1 / 1$ & $100 \% / 100 \% / 100 \%$ & $\begin{array}{c}1 / 1 / 1 \\
0.77 / 0.89 /\end{array}$ \\
\hline Knee Extension & $25(20.8 \%)$ & $87 \% / 87 \%$ & $0.63 / 0.55$ & $95 \% / 95 \% / 95 \%$ & 0.83 \\
\hline \multicolumn{6}{|l|}{ Follow Through } \\
\hline Arm Across Body & $2(1.7 \%)$ & $97 \% / 97 \%$ & $-0.01 /-0.01$ & $100 \% / 90 \% / 100 \%$ & $1 /-0.05 / 1$ \\
\hline Trunk Flexion & $25(20.8 \%)$ & $70 \% / 70 \%$ & $0.16 / 0$ & $100 \% / 70 \% / 95 \%$ & $1 / 0.38 / 0.86$ \\
\hline \multicolumn{6}{|l|}{ Overall Impression } \\
\hline Excellent & $20(16.7 \%)$ & & & & \\
\hline Average & $84(70 \%)$ & $63 \% / 67 \%$ & $0.18 / 0.31$ & $100 \% / 85 \% / 95 \%$ & $1 / 0.57 / 0.90$ \\
\hline Poor & $16(13.3 \%)$ & & & & \\
\hline
\end{tabular}

Table 1. Evaluation of inter- and intra-rater reliability for each item in the ACES scoring tool.

ternal rotation, shoulder abduction), two arm cocking phase variables (glove arm, maximum shoulder external rotation), and two ball release variables (lateral trunk tilt, shoulder abduction).

In an unpublished study (E. Quatromoni, unpublished data, 2015), Quatromoni evaluated 34 adolescent baseball pitchers using high-speed 2D video to assess the intra- and inter-rater reliability of six biomechanical errors [forearm position at stride foot contact (SFC), stride foot position, backward lean at SFC, shoulder position at SFC, trunk to elbow angle at SFC, and contralateral trunk lean at maximal humeral external rotation] of three pitches (trials) analyzed independently by three raters (one former collegiate pitcher and two certified athletic trainers). Regarding intra-rater reliability across the three trials, five of the six biomechanical error items had fair to excellent agreement, producing kappa values between 0.459 and 0.872 , $\mathrm{p}<0.05$. Only open shoulder position at SFC (specifically trial 2 vs. 3) had poor agreement $(\mathrm{kappa}=0.242, \mathrm{p}>0.05)$. Inter-rater reliability of the six biomechanical errors demonstrated mixed results, with three out of six (50\%) variables having fair to good agreement $(\mathrm{kappa}=0.518$ to $0.580, \mathrm{p} \leqslant 0.001)$. In the current study involving 20 adolescent baseball pitchers, intra-rater reliability across the two trials was fair to excellent, with all 20 items in the checklist producing kappa between 0.47 and 1. Inter-rater reliability for the ACES scoring tool was fair to excellent (kappa $=0.44$ to 1 ) on 10 out of $20(50 \%)$ variables: three windup phase variables (center of gravity, knee height, lead hip), three stride foot contact variables (external rotation at SFC, stride length, and trunk rotation), two acceleration variables (trunk flexion, knee flexion) and two deceleration variables (arm internal rotation, knee extension). Because these 10 variables had acceptable intraand inter-rater reliability, these 10 items may be considered key components of a scoring system that can be utilized for identifying impaired pitching mechanics.

Davis et al. ${ }^{14}$ evaluated 169 baseball pitchers aged 9 to 18 years using 2D video analysis and 3D motion analysis to determine if correct performance of five biomechanical pitching parameters considered to be key elements in youth 


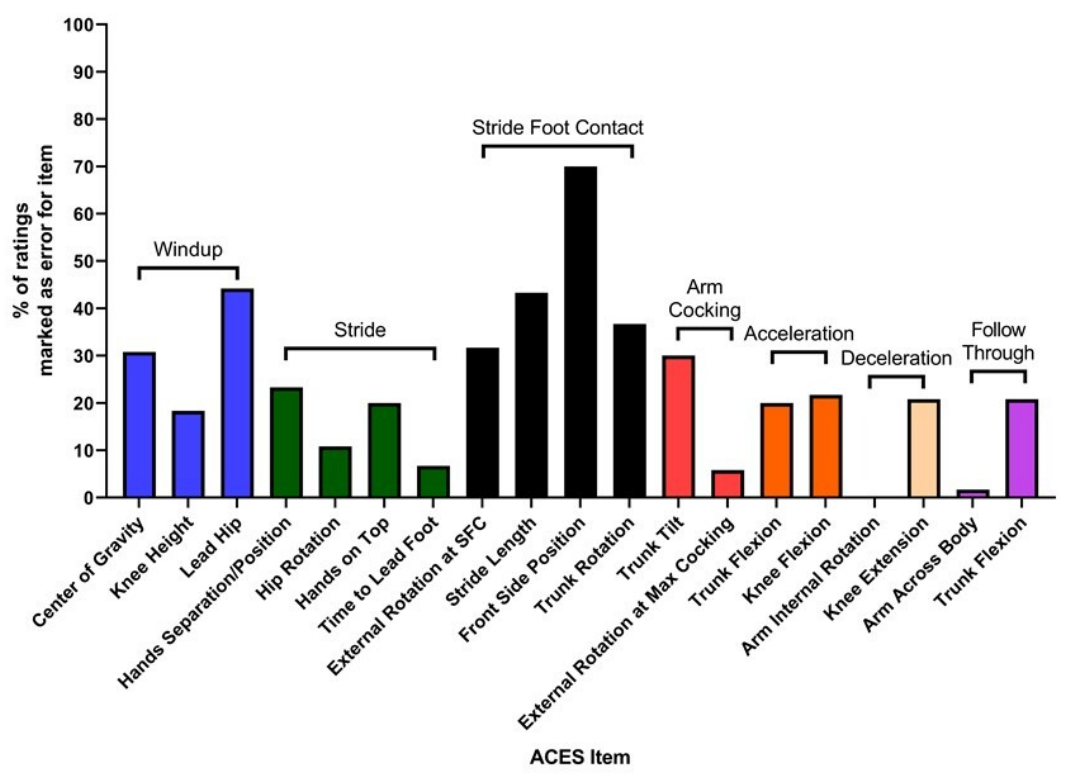

Figure 2. Relationship Between Individual ACES Scoring Tool Item, Phase of Throwing, and Rater-Identified Errors

pitching (leading with the hips, hand-on-top position, arm in throwing position, closed-shoulder position, and stride foot toward home plate) were associated with decreased joint forces at the shoulder and elbow (humeral internal rotation torque [HIRT] and elbow valgus load [EVL]). Interestingly, pitchers that performed both the hand-on-top and closed-shoulder positions correctly were considered to be more efficient than those pitchers who performed these parameters incorrectly. Several ACES items (items 3, 6, 10) were modeled after the parameters of this study.

Regarding individual ACES items (Figure 2), the authors found that one windup phase variable (leading with the hips) and four stride foot contact phase variables (semicocked flexed elbow, abducted and externally rotated shoulder-represented as External Rotation at SFC in Table 1 and Figure 2; stride length; front side position; and trunk rotation) were responsible for 271 out of 548 (49.5\%) total errors identified by raters (Table 1). Fifty-three of 120 (44.2\%) ratings in the current study identified adolescent pitchers leading with the hips (premature forward momentum). In a descriptive laboratory study, Davis et al. ${ }^{14}$ found that 77 of 83 (92.8\%) adolescent pitchers demonstrated "leading with the hips" as well. Surprisingly, "leading with the hips" was associated with higher HIRT, higher elbow EVL, and lower pitching efficiency (efficiency defined as normalized HIRT/ velocity and normalized EVL/velocity. These ratios indicate the amount of stress the shoulder and elbow are subjected to for a given pitch velocity generated; the higher the value, the lower the efficiency). While increased forces ( $\uparrow$ HIRT, $\uparrow$ EVL) cannot be considered causal to increased risk of injury, ${ }^{14}$ several authors have suggested that increased HIRT is a contributor to a physiological condition of proximal humeral epiphysiolysis (Little League Shoulder), as well as humeral retrotorsion, a physiologic adaptation to the proximal humerus that can contribute to increased external rotation and increased velocity. ${ }^{25}$ While "leading with the hips" may be necessary for generating ball velocity in youth and adolescent pitchers, ${ }^{25-27}$ it remains unclear whether this parameter should be promoted by pitching coaches since the potential long-term risks (cumulative microtrauma due to increased kinetic forces at the shoulder and elbow) may outweigh transient performance benefits (velocity) in skeletally and physiologically immature pitchers. Consequently, "leading with the hips" was categorized as a biomechanical error rather than a correct parameter in this study.

The culminating event that ends the stride phase (Appendix III), stride foot contact (eg., when the lead foot contacts the ground), has significant biomechanical implications that optimally allow transfer of energy to the distal segments of the kinetic chain. Aberrations in stride length, lead shoulder position (eg, "open” vs. "closed" shoulder position), and stride foot contact position have been associated with increased shoulder and elbow forces ${ }^{28}$ as well as decreases in ball velocity, which can result in greater demands on the distal kinetic chain to maintain throwing accuracy and velocity. ${ }^{22,29}$ Stride errors in direction and length can affect trunk rotation velocity and inclination (contralateral trunk tilt) and can lead to the throwing arm lagging behind the scapular plane, which is a known contributor to increased stress on the shoulder and elbow. ${ }^{30}$ The results of this study demonstrate that ACES items categorized as stride foot contact variables collectively accounted for the largest percentage of erroneous ratings, perhaps identifying stride foot contact as the most important biomechanical event to objectively assess in adolescent pitchers. 


\section{LIMITATIONS}

This study had several limitations. While the ACES tool was constructed by those with backgrounds in researching baseball biomechanics, the contribution of each variable to pitching, the kinetic chain, and overall injury risk remains unclear. Additionally, some components of the ACES tool required that several conditions be met to be graded as "correct," such as item 10 that consisted of three variables for a correct score; this may have increased error in grading the throwing motion.

This small sample of adolescent pitchers likely represented more frequent pitching inefficiencies compared to higher level pitchers; however, adolescents are the largest subset of pitchers in organized baseball, with more than 6 million adolescents participating in organized baseball in the United States. ${ }^{18}$ Compared to elite-level pitchers, adolescent pitcher biomechanical studies have been underrepresented in the literature. Single-episode study design $12,14,16,18,24,25$ and studies using only one pitch for 2D video analysis ${ }^{15}$ (including this study) limit the ability to assess for trends, including correction of biomechanical errors and development of mechanical inefficiency with fatigue in pitchers' biomechanics. Additionally, the ACES tool may not be generalizable to pitchers with different throwing mechanics which are widely regarded as "non-traditional" (ie, side-arm, or submarine pitchers).

As this study was conducted at two clinical research centers, cameras with different speeds (300 and 120 frames per second) were utilized. While not uniform, these frame rates are within the range of commercial equipment available to the consumer, including coaches, parents, and players. Additionally, the use of a catcher or target was not uniform at the two clinical research centers, as one center standardly used a staff member experienced with catching pitchers during $2 \mathrm{D}$ video analysis, while the other center utilized a strike zone target. Lastly, non-healthcare providers were not utilized (eg, youth baseball coach) as a rater in this study, limiting the ability to determine the usefulness of the ACES scoring system for a layperson without specialized training in interpretation of adolescent pitching biomechanics.

\section{CONCLUSION}

The results of this study indicate that $2 \mathrm{D}$ video analysis and an observational screening tool (ACES) to assess pitching kinematics can be used reliably, due to excellent intra- and inter-rater reliability demonstrated by raters of different musculoskeletal disciplines (orthopedic surgeon, physical therapist, certified athletic trainer). Future studies validating 2D video analysis methodology with 3D motion analysis are warranted before the ACES screening tool is disseminated and employed by coaches and instructors of adolescent pitchers.

\section{FINANCIAL DISCLOSURE}

The authors have no applicable financial disclosures.

\section{CONFLICTS OF INTEREST}

The authors declared that they have no conflicts of interest in the authorship and publication of this contribution.

Submitted: January 31, 2021 CST, Accepted: August 05, 2021 CST 


\section{REFERENCES}

1. Carson WG, Gasser SI. Little Leaguer's Shoulder: a report of 23 cases. Am J Sport Med. 1998;26(4):575-580. doi:10.1177/03635465980260041 $\underline{901}$

2. Braun S, Kokmeyer D, Millett PJ. Shoulder injuries in the throwing athlete. J Bone Joint Surg - A. 2009;91(4):966-978. doi:10.2106/JBJS.H.01341

3. Popchak A, Burnett T, Weber N, Boninger M. Factors related to injury in youth and adolescent baseball pitching, with an eye toward prevention. $\mathrm{Am}$ J Phys Med Rehabil. 2015;94(5):395-409. doi:10.1097/ PHM.0000000000000184

4. Rossy WH, Oh LS. Pitcher's elbow: medial elbow pain in the overhead-throwing athlete. Curr Rev Musculoskelet Med. 2016;9(2):207-214. doi:10.1007/s1 2178-016-9346-7

5. Fleisig GS, Andrews JR. Prevention of elbow injuries in youth baseball pitchers. Sports Health. 2012;4(5):419-424. doi:10.1177/1941738112454828

6. Osbahr DC, Chalmers PN, Frank JS, Williams RJ, Widmann RF, Green DW. Acute, avulsion fractures of the medial epicondyle while throwing in youth baseball players: A variant of Little League elbow. J Shoulder Elb Surg. 2010;19(7):951-957. doi:10.1016/j.j $\underline{\text { se.2010.04.038 }}$

7. Baker CL, Romeo AA, Baker CL. Osteochondritis dissecans of the capitellum. Am J Sports Med. 2010;38(9):1917-1928. doi:10.1177/036354650935496 $\underline{9}$

8. Nassab PF, Schickendantz MS. Evaluation and treatment of medial ulnar collateral ligament injuries in the throwing athlete. Sports Med Arthrosc. 2006;14(4):221-231. doi:10.1097/01.jsa.0000212323.3 8807.fa

9. Melugin HP, Leafblad ND, Camp CL, Conte S. Injury prevention in baseball: from youth to the pros. Curr Rev Musculoskelet Med. 2018;11(1):26-34. doi:10.100 7/s12178-018-9456-5

10. Shitara H, Yamamoto A, Shimoyama D, et al. Shoulder stretching intervention reduces the incidence of shoulder and elbow injuries in high school baseball players: a time-to-event analysis. $\mathrm{Sci}$ Rep. 2017;7(October 2016):1-7. doi:10.1038/srep4530 $\underline{4}$
11. Erickson BJ, Sgori T, Chalmers PN, et al. The impact of fatigue on baseball pitching mechanics in adolescent male pitchers. Arthroscopy. 2016;32(5):762-771. doi:10.1016/j.arthro.2015.11.051

12. Fleisig GS, Barrentine SW, Zheng N, Escamilla RF, Andrews JR. Kinematic and kinetic comparison of baseball pitching among various levels of development. J Biomech. 1999;32(12):1371-1375. do i:10.1016/S0021-9290(99)00127-X

13. Fleisig GS, Barrentine SW, Escamilla RF, Andrews JR. Biomechanics of overhand throwing with implications for injuries. Sport Med. 1996;21(6):421-437. doi:10.2165/00007256-19962106 $\underline{0-00004}$

14. Davis JT, Limpisvasti O, Fluhme D, et al. The effect of pitching biomechanics on the upper extremity in youth and adolescent baseball pitchers. Am J Sports Med. 2009;37(8):1484-1491. doi:10.1177/0 $\underline{363546509340226}$

15. Sgroi T, Chalmers PN, Riff AJ, et al. Predictors of throwing velocity in youth and adolescent pitchers. $J$ Shoulder Elb Surg. 2015;24(9):1339-1345. doi:10.1016/ j.jse.2015.02.015

16. Okoroha KR, Lizzio VA, Meta F, Ahmad CS, Moutzouros V, Makhni EC. Predictors of elbow torque among youth and adolescent baseball pitchers. Am J Sport Med. 2018;46(9):2148-2153. doi:10.1177/036354 $\underline{6518770619}$

17. Nissen CW, Westwell M, Ounpuu S, et al. Adolescent baseball pitching technique: A detailed three-dimensional biomechanical analysis. Med Sci Sports Exerc. 2007;39(8):1347-1357. doi:10.1249/mss.0 b013e318064c88e

18. Nissen CW, Westwell M, Õunpuu S, Patel M, Solomito M, Tate J. A biomechanical comparison of the fastball and curveball in adolescent baseball pitchers. Am J Sport Med. 2009;37(8):1492-1498. doi:1 $\underline{0.1177 / 0363546509333264}$

19. Erickson BJ, Nwachukwu BU, Rosas S, et al. Trends in medial ulnar collateral ligament reconstruction in the United States: A retrospective review of a large private-payer database from 2007 to 2011. Am J Sport Med. 2015;43(7):1770-1774. doi:10.1177/0363546515 $\underline{580304}$

20. Padaki AS, Ahmad CS. Can we reduce the epidemic of elbow injuries in youth throwers? J Orthop Sports Phys Ther. 2018;48(5):354-357. doi:10.2 519/jospt.2018.0607 
21. De Froda SF, Thigpen CA, Kriz PK. Twodimensional video analysis of youth and adolescent pitching biomechanics: A tool for the common athlete. Curr Sports Med Rep. 2016;15(5):350-358. do i:10.1249/JSR.0000000000000295

22. Cohen J. A coefficient of agreement for nominal scales. Educ Psychol Meas. 1960;20:37-46.

23. Mandrekar JN. Measures of interrater agreement. J Thorac Oncol. 2011;6(1):6-7. doi:10.1097/JTO.0b013e3 $\underline{18200 f 983}$

24. Nicholls R, Fleisig G, Elliott B, Lyman S, Osinski E. Accuracy of qualitative analysis for assessment of skilled baseball pitching technique. Sports Biomech. 2003;2(2):213-226. doi:10.1080/14763140308522819

25. Sabick MB, Kim Y-K, Torry MR, Keirns MA, Hawkins RJ. Biomechanics of the shoulder in youth baseball pitchers: implications for the development of proximal humeral epiphysiolysis and humeral retrotorsion. Am J Sports Med. 2005;33(11):1716-1722. doi:10.1177/03635465052753 $\underline{47}$
26. Sabick MB, Torry MR, Kim Y-K, Hawkins RJ. Humeral torque in professional baseball pitchers. $A m$ J Sports Med. 2004;32(4):892-898.

27. Keeley DW, Hackett T, Keirns M, Sabick MB, Torry MR. A biomechanical analysis of youth pitching mechanics. J Pediatr Orthop. 2008;28(4):452-459. do i:10.1097/BPO.0b013e31816d7258

28. Fortenbaugh D, Fleisig GS, Andrews JR. Baseball pitching biomechanics in relation to injury risk and performance. Sports Health. 2009;1(4):314-320. doi:1 $\underline{0.1177 / 1941738109338546}$

29. Weber AE, Kontaxis A, O’Brien SJ, Bedi A. The biomechanics of throwing: Simplified and cogent. Sports Med Arthrosc. 2014;22(2):72-79. doi:10.1097/IS A.0000000000000019

30. Calabrese GJ. Pitching mechanics, revisited. Int J Sports Phys Ther. 2013;8:652-660. 


\section{SUPPLEMENTARY MATERIALS}

\section{Appendix 1}

Download: https://ijspt.scholasticahq.com/article/29869-reliability-of-an-observational-biomechanical-analysis-toolin-adolescent-baseball-pitchers/attachment/75413.pdf

\section{Appendix 2}

Download: https://ijspt.scholasticahq.com/article/29869-reliability-of-an-observational-biomechanical-analysis-toolin-adolescent-baseball-pitchers/attachment/75860.pdf

\section{Appendix 3}

Download: https://ijspt.scholasticahq.com/article/29869-reliability-of-an-observational-biomechanical-analysis-toolin-adolescent-baseball-pitchers/attachment/75861.pdf 